\title{
Efeito do volume do recipiente na produção de mudas e no crescimento inicial de Jatropha curcas L. no Oeste Paranaense
}

\section{The effect of container volume on seedling production and initial growth of Jatropha curcas L. on the Western Parana}

\author{
Michelle Cristina Ajala ${ }^{1}$; Noelle Farias de Aquino ${ }^{2}$; \\ Ubirajara Contro Malavasi ${ }^{3^{*}}$; Marlene de Matos Malavasi ${ }^{3}$
}

\begin{abstract}
Resumo
Este trabalho objetivou comparar a influência do volume de recipientes na produção de mudas e no crescimento, durante o primeiro ano, de pinhão manso no oeste paranaense. A produção de mudas no viveiro utilizou saco plástico de $1.178 \mathrm{~cm}^{3}$ e tubetes de 120 e $180 \mathrm{~cm}^{3}$ preenchidos com substrato comercial. As mensurações quinzenais incluíram os incrementos em altura, diâmetro do coleto e área foliar. Após o plantio foram avaliados os incrementos no número de folhas, de ramificações e a porcentagem de sobrevivência trimestral. Ao final da fase de viveiro, o maior incremento em altura $(1,56 \mathrm{~cm})$ e em diâmetro do coleto $(1,3 \mathrm{~mm})$ foi obtido nas mudas de $J$. curcas conduzidas em saco plástico com volume de $1.178 \mathrm{~cm}^{3}$, enquanto que os valores para mudas em tubetes de $180 \mathrm{~cm}^{3} \mathrm{e}$ $120 \mathrm{~cm}^{3}$ resultaram em 1,24 cm e $1,2 \mathrm{~mm}$ e $1,48 \mathrm{~cm}$ e 1,1 mm, respectivamente. Após o plantio, não foram verificadas diferenças nos incrementos trimestrais em altura, diâmetro de colo, número de folhas, número de ramos (primários e secundários) e porcentagem de mortalidade em função do volume dos recipientes utilizados para a formação das mudas. Os incrementos observados no primeiro trimestre após o plantio apresentaram os maiores valores em diâmetro do coleto, número de folhas, e mortalidade. Mudas de J.curcas semeadas no final de agosto, conduzidas em viveiro de setembro a novembro em tubetes de $120 \mathrm{~cm}^{3}$, e plantadas em dezembro em solo PVd da região oeste do Estado do Paraná resultam em crescimento equivalente ao de mudas produzidas em recipientes de maior volume com economia de substrato, espaço de viveiro e esforço no plantio.
\end{abstract}

Palavras-chave: Pinhão manso, tubete, viveiro florestal, morfometria

\begin{abstract}
This study compares the influence of the volume of containers in seedling production and growth during the first year of Jatropha in western Paraná. The production of seedlings in the nursery used plastic bags of $1,178 \mathrm{~cm}^{3}$ and containers of $120 \mathrm{~cm}^{3}$ and $180 \mathrm{~cm}^{3}$ filled with commercial substrate. Measurements included increments in height, stem diameter and leaf area. After planting, measurements every three months included the increase in number of leaves, branches, and the survival percentage. At the end of the nursery production phase the largest increase in height $(1.56 \mathrm{~cm})$ and stem diameter $(1.3 \mathrm{~mm})$ were measured in seedlings of $J$. curcas conducted in $1,178 \mathrm{~cm}^{3}$ plastic bags, whereas the values calculated for seedlings from other containers resulted in values of $1.24 \mathrm{~cm}$ and $1.2 \mathrm{~mm}$ from $180 \mathrm{~cm}^{3}$ plugs, and $1.48 \mathrm{~cm}$ and $1.1 \mathrm{~mm}$ from $120 \mathrm{~cm}^{3}$ plugs. After planting, there were no differences in the increments in height, stem diameter, leaf number, number of branches (primary and secondary) and mortality rate due to container volume. The increments obtained in the first quarter after planting showed higher values

1 Doutoranda em Agronomia, Universidade Estadual do Oeste do Paraná, UNIOESTE, Marechal Cândido Rondon, PR. E-mail: michelleajala@hotmail.com

2 Mestre em Agronomia, UNIOESTE, Marechal Cândido Rondon, PR. E-mail: noellebio@yahoo.com.br

3 Profs. da UNIOESTE, Marechal Cândido Rondon, PR. E-mail: biramalavasi@yahoo.com.br; malavasi@unioeste.br
\end{abstract}

Autor pra correspondência 
for stem diameter, leaf number, and mortality. J.curcas seedlings sown in late August and conducted in the nursery from September to November in containers of $120 \mathrm{~cm}^{3}$, and planted in December in a dystrophic red alfisol in western Paraná resulted in growth equivalent to that of seedlings grown in containers with greater volume with saving of substrate, space and efforts in the nursery.

Key words: Physic nut, plug, forest nursery, morphometry

\section{Introdução}

O pinhão manso contribui na questão de fontes alternativas para a produção de energia, biodiesel e fármacos (GINWAL et al., 2005). As sementes dessa espécie encontram-se entre as oleaginosas mais promissoras do Brasil, por possuir alto teor de óleo, fácil cultivo e variações pouco significativas de acidez (TAPANES; ARANDA; CARNEIRO, 2006), porém com germinação muito variável dependendo da geografia e de fatores ambientais (GINWAL et al., 2005; SATURNINO et al., 2005).

A produtividade do pinhão manso varia muito em função da região de plantio, idade da cultura e método de cultivo, e tratos culturais. Entretanto, outros fatores são considerados como a quantidade de chuva e a fertilidade do solo (ARRUDA et al., 2004). A espécie pode ser implantada em plantio solteiro ou consorciada através da semeadura direta ou com uso de mudas. A escolha do sistema dependerá exclusivamente da finalidade da cultura, dos objetivos do agricultor e das condições locais (SATURNINO et al., 2005).

$\mathrm{Na}$ produção de mudas, o tipo de sistema de irrigação a ser utilizado e sua capacidade de produção anual são determinados a partir da escolha do recipiente (FERRARI, 2003). Gomes, Couto e Borges (1990) e Viana et al. (2008) relataram a importância de estudar as dimensões e os volumes dos recipientes para a produção de mudas, salientando que recipientes maiores que os recomendáveis resultam em custos desnecessários de substrato.

A escolha do melhor recipiente a ser utilizado deve levar em consideração a quantidade de mudas a serem produzidas e o tempo que estas permanecerão no viveiro (SILVA; STEIN, 2008). Outro importante fator na produção de mudas de alta qualidade morfo-fisiológica consiste no método como estas são produzidas (SCHIAVO; MARTINS, 2003). O tamanho do recipiente influencia diversas características da muda, a taxa de sobrevivência à campo, e a produtividade da cultura. O tamanho e a forma do recipiente influenciam no crescimento e desenvolvimento tanto da raiz como da parte aérea (SOUZA, 1995). Os sacos plásticos apresentam maior disponibilidade no mercado, menor custo de aquisição e baixo investimento em infra-estrutura de viveiros, sendo utilizados pelos viveiristas que produzem pequenas quantidades de mudas. No entanto, a implantação de extensas áreas com plantio de espécies lenhosas estimulou o uso de recipientes (tubetes) que permitam a mecanização e a formação de mudas em larga escala (CAMPINHOS JUNIOR; IKEMORI, 1983).

Os recipientes de melhor aceitação no mercado são os tubetes, pois apresentam inúmeras vantagens. Dentre eles destacam-se ocupar uma menor área no viveiro, permitir o acondicionamento de um número grande de mudas, possibilitar a automatização desde o enchimento até a semeadura e a expedição, e são reutilizáveis (FERRARI, 2003).

Recipientes de paredes rígidas podem comportar uma pequena quantidade de substrato podendo provocar deformações no sistema radicular, refletindo no crescimento e desenvolvimento da parte aérea das mudas, os quais persistem no campo (BARROSO, 1999). Entretanto, deve haver um ponto de equilíbrio entre o volume e o formato do recipiente na escolha destes, visando obter uma muda de qualidade, mas que também minimizem os custos da produção (LIMA et al., 2006).

$\mathrm{Na}$ produção de mudas, os sacos plásticos são bastante utilizados, porém seu uso vem diminuindo devido a grande quantidade de substrato exigida, o 
peso da muda pronta e a ocupação de uma área maior no viveiro, o que diminui a produção por unidade de área. Ainda, há uma maior dificuldade no transporte das mudas e necessidade de mais mão de obra. Entretanto, os sacos plásticos têm como vantagem seu baixo custo, a possibilidade de utilizar sistemas de irrigação simples e a de se obter mudas de maior tamanho (FERRARI, 2003).

Neste contexto, buscou-se testar diferentes recipientes na produção de mudas de pinhão manso, avaliando-se, também, o crescimento inicial durante o primeiro ano de plantio a campo no oeste paranaense.

\section{Material e Métodos}

O ensaio utilizou sementes procedentes de Dom Juan Cabalero (Paraguai) e germinantes originados da germinação em BOD (Fanen ${ }^{\circledR}$ modelo $\left.347 \mathrm{G}\right)$ à temperatura de $25^{\circ} \mathrm{C}$, sem fotoperíodo, em substrato sobre areia auto-clavada a $120^{\circ} \mathrm{C}$ por 15 minutos (HÖRING; MALAVASI; MALAVASI, 2011). Os germinantes foram repicados oito dias após a germinação (protusão da radícula maior que $2 \mathrm{~mm}$ ) para saco plástico com volume de $1.178 \mathrm{~cm}^{3}$ (T1), tubetes de polipropileno com volume de $180 \mathrm{~cm}^{3}$ (T2) ou volume de $120 \mathrm{~cm}^{3}$ (T3) que constituíram os tratamentos. O ensaio utilizou o substrato comercial Plantmax ${ }^{\circledR}$ em todos os recipientes que permaneceram no viveiro protegidos com Sombrite ${ }^{\circledR}(60 \%)$ por 4 semanas. Em seguida, todas as mudas foram expostas a pleno sol durante 8 semanas. Na fase de viveiro, as mudas receberam irrigação via aspersão por 15 minutos duas vezes ao dia sem fertilização de cobertura.

As variáveis analisadas na fase de viveiro incluíram os incrementos em altura (quantificados com régua milimetrada do coleto até a última interseção caulinar) e no diâmetro do coleto (mensurado com paquímetro digital). Nesta fase, o ensaio foi conduzido de acordo com um delineamento de blocos casualizados (DBC) formado por quatro blocos com 20 mudas úteis de cada tratamento (repetições).

O plantio a campo ocorreu 12 semanas após a repicagem em área experimental no município de Pato Bragado (PR) com coordenadas geográficas de $24^{\circ} 37^{\prime} 35^{\prime \prime} \mathrm{S}$ e $54^{\circ} 13^{\prime} 29^{\prime}$ ' W, e altitude de 288 m. O solo da área é classificado como Latossolo Vermelho distrófico (LVd) de textura argilosa (EMBRAPA, 2006), enquanto o clima recebe a classificação de Cfa, com precipitação anual total de $1.500 \mathrm{~mm}$.

O plantio utilizou espaçamento de $3 \times 2 \mathrm{~m}$ com coveamento $(30 \times 30 \times 30 \mathrm{~cm})$ manual. Os tratos culturais constaram de roçadas e coroamento (diâmetro de $1,0 \mathrm{~m}$ ) trimestrais. As variáveis quantificadas na fase pós-plantio incluíram os incrementos em altura, diâmetro do colo, número de folhas, número de bifurcações, número de ramificações, área foliar e mortalidade. A área foliar utilizou imagens analisadas pelo programa QUANT com amostragem conforme preconizado por Vale, Fernades Filho e Liberato (2003) e extrapolada em função do número de folhas. As quantificações acima mencionadas ocorreram na época do plantio (dezembro/2007) e nos meses de março, junho, setembro e dezembro de 2008, resultando em quatro estimativas dos incrementos pós-plantio.

O ensaio a campo utilizou o delineamento de blocos casualizados similar ao utilizado no viveiro totalizando 240 plantas. Os incrementos das variáveis mensuradas tanto na fase de viveiro como aqueles após o plantio foram submetidos ao teste de Barlett e à análise de variância com uso do software SAEG. Quando necessário, compararam-se as médias dos tratamentos com o teste Tukey a 5\%.

\section{Resultados e Discussão}

A análise dos dados na fase de viveiro constatou efeitos significativos $(\mathrm{P}<0,05)$ nos incrementos em altura e diâmetro do coleto das mudas em função das datas de avaliação e dos volumes dos recipientes. O crescimento das mudas de pinhão manso (Tabela 1) enfatizou o crescimento em altura dos 16 aos 30 dias após repicagem (DAR) enquanto o maior incremento no diâmetro do coleto ocorreu dos 46 aos 60 DAR. 
Tabela 1. Incrementos em altura e diâmetro do coleto de mudas de pinhão manso (Jatropha curcas L.) em função dos dias após repicagem (DAR). Santa Helena, PR, 2007.

\begin{tabular}{lcc}
\hline Dias após repicagem (DAR) & \multicolumn{2}{c}{ Incremento } \\
\hline & Altura $(\mathrm{cm})$ & Diâmetro do coleto $(\mathrm{mm})$ \\
1 a 15 & $0,7 \mathrm{ab}$ & $0,8 \mathrm{c}$ \\
16 a 30 & $1,2 \mathrm{a}$ & $1,2 \mathrm{~b}$ \\
31 a 45 & $0,3 \mathrm{~d}$ & $1,1 \mathrm{~b}$ \\
46 a 60 & $0,9 \mathrm{ab}$ & $1,5 \mathrm{a}$ \\
61 a 75 & $0,6 \mathrm{bcd}$ & $0,8 \mathrm{c}$ \\
76 a 90 & $0,3 \mathrm{~d}$ & $0,7 \mathrm{c}$ \\
\hline
\end{tabular}

Médias seguidas da mesma letra na coluna não diferem estatisticamente pelo teste de Tukey a 5\%

Fonte: Elaboração dos autores.

Mudas de pinhão manso formadas em sacos plásticos obtiveram os maiores incrementos em altura e diâmetro do coleto (Tabela 2). Entretanto, mudas formadas em sacos plásticos externaram valores que não diferiram $(\mathrm{P}>0,05)$ daqueles obtidos em tubetes de $120 \mathrm{~cm}^{3}$ com respeito ao incremento em altura, e daqueles obtidos em tubetes de $180 \mathrm{~cm}^{3}$ em relação ao incremento em diâmetro.

Resultados similares foram externados por Paulino et al. (2011), que reportaram inexistir diferenças seis semanas após a emergência ao compararem os efeitos do volume do recipiente (tubetes de $120 \mathrm{~cm}^{3}, 180 \mathrm{~cm}^{3}$ e $290 \mathrm{~cm}^{3}$ e sacos plásticos de $500 \mathrm{~cm}^{3}$ ) na formação de mudas de pinhão manso.
Segundo Pezzutti, Scnumacher e Hoppe (1999), o conhecimento do crescimento das plantas no viveiro, em resposta a fatores como água, luz, temperatura, fertilizantes e restrição radicular reveste-se de importância para produzir mudas de qualidade, em quantidade suficiente, e a um custo menor. Ao final da fase de viveiro, o maior incremento em altura $(1,56 \mathrm{~cm})$ e em diâmetro do coleto $(1,3 \mathrm{~mm})$ foi calculado nas mudas de $J$. curcas conduzidas em saco plástico com volume de $1.178 \mathrm{~cm}^{3}$. Os incrementos para mudas produzidas em tubetes de $180 \mathrm{~cm}^{3}$ e $120 \mathrm{~cm}^{3}$ resultaram em 1,24 $\mathrm{cm}$ e $1,2 \mathrm{~mm}$ e $1,48 \mathrm{~cm}$ e $1,1 \mathrm{~mm}$, respectivamente para os incrementos em altura e diâmetro do coleto (Tabela 2).

Tabela 2. Incrementos em altura e coleto de Jatropha curcas L. em função do volume dos recipientes. Santa Helena, PR, 2007.

\begin{tabular}{lll}
\hline Tratamento & Altura $(\mathrm{cm})$ & Coleto $(\mathrm{mm})$ \\
\hline Sacos plásticos $1.178 \mathrm{~cm}^{3}$ & $1,56 \mathrm{a}$ & $1,27 \mathrm{a}$ \\
Tubete $180 \mathrm{~cm}^{3}$ & $1,24 \mathrm{~b}$ & $1,22 \mathrm{a}$ \\
Tubete $120 \mathrm{~cm}^{3}$ & $1,48 \mathrm{a}$ & $1,08 \mathrm{~b}$ \\
\hline
\end{tabular}

Médias seguidas da mesma letra na coluna não diferem estatisticamente pelo teste de Tukey a 5\%

Fonte: Elaboração dos autores.

Os resultados em relação à formação da parte aérea assemelham-se aos de Avelar et al. (2006) na produção de mudas de pinhão manso em tubetes de $50 \mathrm{~cm}^{3}$ e $120 \mathrm{~cm}^{3}$ em Minas Gerais. A obtenção de mudas de pinhão manso de maiores dimensões com a utilização de recipientes de maior volume possui similaridade nos trabalhos de Oliveira et al. (2010) que relataram maior produção de matéria seca aérea e radicular em mudas de pinhão manso conduzidas em sacos plásticos de $5.000 \mathrm{~cm}^{3}$ a pleno 
sol nas condições de Areia (PB), e de Severino, Lima e Beltrão (2007) que recomendaram o saco plástico com volume de $2.000 \mathrm{~cm}^{3}$ nas condições de Campina Grande (PB). Similarmente, Nicoloso et al. (2000) reportaram maiores incrementos em altura, diâmetro e porcentagem de sobrevivência quando da utilização de sacos plásticos com volume de $3.064 \mathrm{~cm}^{3}$ na produção de mudas de Maytenus ilicifolia e Apuleia leiocarpa aos 120 DAE.

Viana et al. (2008) mencionaram que o tamanho ideal do recipiente para produção de mudas dependerá do ritmo de crescimento das plantas, o qual é função da espécie e das condições de clima e substrato. Ao final da fase de viveiro, as mudas de pinhão manso atingiram em média $12,4 \mathrm{~cm}, 9,3$ cm e $12,2 \mathrm{~cm}$ de altura e $9,3 \mathrm{~mm}, 8,5 \mathrm{~mm}$ e $8,0 \mathrm{~mm}$ de diâmetro do coleto quando conduzidas em saco plástico de $1.178 \mathrm{~cm}^{3}$, tubete de $180 \mathrm{~cm}^{3}$ e de 120 $\mathrm{cm}^{3}$, respectivamente.

A análise dos dados coletados pós-plantio indicou inexistirem $(\mathrm{P}>0,05)$ diferenças nos incrementos trimestrais em altura, diâmetro de colo, número folhas, número de ramos (primários e secundários) e na porcentagem de mortalidade em função do volume do recipiente utilizado para a formação das mudas. Contrastando com os resultados obtidos neste ensaio, Souza, Freire e Sampaio (2009) reportaram que mudas de pinhão manso produzidas em recipientes de 2,1 e 3,9 L de volume apresentaram maior crescimento em altura e no diâmetro do colo seis meses após o plantio nas condições de Porto Velho, RO. Semelhantemente, Leles et al. (2000) com Eucalyptu e Viana et al. (2008) com Bauhinia forficata reportaram a influência positiva do volume do recipiente no desenvolvimento das mudas após plantio a campo. No entanto, Malavasi e Malavasi (2006) com mudas de Cordia trichotoma e Jacaranda micranta na mesma região do presente ensaio, e José, Davide e Oliveira (2005) com mudas Schinus terebinthifolius em área degradada pela exploração da bauxita externaram resultados similares aos aqui verificados.
O pinhão manso, por ser uma planta asselvajada apresenta significativa desuniformidade quanto a crescimento, arquitetura e desenvolvimento (ALBUQUERQUE et al., 2009). A morfometria do pinhão manso apresenta diferenças fortemente influenciadas pela pluviosidade, adensamento de plantio, adubação, variabilidade arquitetônica, peculiaridades na caducifólia que varia entre as plantas e ambientes de cultivo como solo e clima (AVELAR et al., 2008).

Dentre os incrementos morfométricos trimestrais, os calculados no primeiro trimestre após o plantio apresentaram os maiores valores em diâmetro do coleto, número de folhas e mortalidade. Os incrementos em altura foram independentes da época de avaliação com um incremento médio de $24,35 \mathrm{~cm}$ por trimestre (Tabela 3 ).

O pinhão-manso possui desenvolvimento tipo cimeira com crescimento exponencial, sendo que cada ramo culmina numa inflorescência que dá origem a dois a três novos ramos. Portanto, quanto maior o número de ramos basais maior será o número de inflorescências nos módulos de crescimento. $\mathrm{O}$ aumento no número de ramificações iniciou no segundo trimestre após o plantio para as primárias, e no terceiro para as secundárias (Tabela 3 ).

Durante o primeiro trimestre após o plantio, a precipitação pluviométrica acumulada totalizou $385 \mathrm{~mm}$, beneficiando o crescimento vegetal das mudas, apesar do maior percentual de mortalidade contabilizado. $\mathrm{O}$ incremento médio em diâmetro e no número de folhas nas mudas de pinhão manso nos primeiros 90 dias após o plantio foram maiores do que os mensurados por Lang (2008) em mudas de Tabebuia avellanedae (5,6 mm e 88,6 folhas) e Anadenanthera colubrina (4,53 mm e 31,6 folhas) nas mesmas condições edafo-climáticas. No entanto, o maior crescimento em diâmetro das mudas de pinhão manso não resultou em menor mortalidade, quando comparado com as reportadas pelo autor acima com mudas de $T$. avellanedae $(2,35 \%)$ e $A$. columbrina $(3,75 \%)$. 
Tabela 3. Incrementos trimestrais de algumas variáveis morfométricas e mortalidade de pinhão manso (Jatropha curcas L.) durante o primeiro ano de crescimento. Pato Bragado, PR, 2008.

\begin{tabular}{lcccc}
\hline \multirow{2}{*}{ Incrementos } & \multicolumn{4}{c}{ Trimestre após plantio } \\
\cline { 2 - 5 } & 1 & 2 & 3 & 4 \\
\hline Altura $(\mathrm{cm})$ & $29,9 \mathrm{~b} \mathrm{a}$ & $25,9 \mathrm{a}$ & $28,2 \mathrm{a}$ & $21,4 \mathrm{a}$ \\
Diâmetro do colo (mm) & $16,3 \mathrm{a}$ & $10,6 \mathrm{ab}$ & $10,8 \mathrm{ab}$ & $6,8 \mathrm{~b}$ \\
Número de ramos primários & $0,5 \mathrm{c}$ & $0,9 \mathrm{~b}$ & $1,9 \mathrm{a}$ & $1,8 \mathrm{a}$ \\
Número de ramos secundários & $0 \mathrm{~b}$ & $0,5 \mathrm{a}$ & $0,6 \mathrm{a}$ & $0,5 \mathrm{a}$ \\
Número de folhas & $92,5 \mathrm{a}$ & $-24,1 \mathrm{c}^{*}$ & $-0,9 \mathrm{bc}$ & $12,4 \mathrm{~b}$ \\
Mortalidade (\%) & $9,2 \mathrm{a}$ & $1,6 \mathrm{~b}$ & $0,7 \mathrm{c}$ & $0,1 \mathrm{c}$ \\
\hline
\end{tabular}

Médias seguidas da mesma letra na linha não diferem estatisticamente pelo teste de Tukey a 5\%. * valores negativos refletem efeito de geada e granizo em 07/08/2008.

Fonte: Elaboração dos autores.

A redução no número de folhas decorreu de geada seguida de granizo, em agosto de 2008. Andrade et al. (2008) investigando os efeitos de temperaturas mínimas no crescimento do pinhão manso afirmaram ocorrer danos foliares devido à baixas temperaturas, mas que a presença de gemas dormentes mais tolerantes que os tecidos foliares proporcionam certa tolerância aquele estresse ambiental.

A estimativa da área foliar em plantas de pinhão manso produzidas em sacos plásticos apresentaram a maior área foliar $(\mathrm{P}<0,05)$ do que as de plantas oriundas de mudas produzidas em tubetes. Os valores calculados nas avaliações realizadas em junho e em dezembro de 2008 ou seja aos 6 e 12 meses após plantio a campo foram de $79,8 \mathrm{~cm}^{2} \mathrm{e}$ $100,6 \mathrm{~cm}^{2}$, respectivamente.

Em mamona, o volume do recipiente influenciou a área foliar e a massa seca aérea e das raízes (LIMA et al., 2006). No mesmo argumento Carvalho Filho, Arrigoni-Blank e Blank (2004) também concluíram que o volume do recipiente influenciou positivamente o número de folhas quando da produção de mudas de Andira fraxinifolia como ocorrido no presente trabalho.

\section{Conclusão}

Mudas de $J$. curcas semeadas no final de agosto, conduzidas em viveiro de setembro a novembro em tubetes de $120 \mathrm{~cm}^{3}$ e plantadas em dezembro em solo LVd da região oeste do Estado do Paraná resultam em crescimento equivalente ao de mudas produzidas em recipiente de maior volume com economia de substrato, espaço de viveiro, e esforço no plantio.

\section{Agradecimentos}

Ao CNPq pela bolsa de estudo e apoio financeiro à pesquisa.

\section{Referências}

ALBUQUERQue, CASTRO, N. H. A.; BELTRÃO, N. E. M.; LUCENA, A. M. A.; ANDRADE, G. A.; CARAMORI, P. H.; SOUZA, F. S.; MARUR, C. J.; RIBEIRO, A. M. A. Temperatura mínima letal para plantas jovens de pinhão manso. Bragantia,Campinas, v. 67, n. 3, p. 799-803, 2008.

ANDRADE, G. A.; CARAMORI, P. H.; SOUZA, F. S.; MARUR, C. J.; RIBEIRO, A. M. A. Temperatura mínima letal para plantas jovens de pinhão manso. Bragantia,Campinas, v. 67, n. 3, p. 799-803, 2008.

ARRUDA, F. P.; BELTRÃO, N. E. M.; ANDRADE, A. P.; PEREIRA, W. E.; SEVERINO, L. S. Cultivo de pinhão manso (Jatropha curcas L.) como alternativa para o semi-árido nordestino. Revista Brasileira de Oleaginosas e Fibrosas, Campina Grande, v. 8, n. 1, p. 789-799, 2004.

AVELAR, R. C.; SILVA, F. M.; CASTRO NETO, P.; FRAGA, A. C. Avaliação do desenvolvimento de pinhão manso (Jatropha curcas L.) do Banco de Germoplasma 
de UFLA, In: CONGRESSO BRASILEIRO DE PLANTAS OLEAGINOSAS, ÓLEOS, GORDURAS E BIODIESEL, 5.; CLÍNICA TECNOLÓGICA EM BIODIESEL, 2., 2008, Lavras. Anais... Lavras: UFLA, 2008. p. 2796-2801.

AVELAR, R. C.; DEPERON JUNIOR, M. A.; CARVALHO, J. P. F.; DOURADO, D. C.; DANFA, S.; QUINTILIANO, A.A.; FRAGA, A.C.; CASTRO NETO, P. Produção de mudas de pinhão manso (Jatropha curcas L.) em tubetes. Minas Gerais: UFLA, 2006. Disponível em: $<$ www.biodiesel.gov.br/docs/congresso2006/ agricultura/produçaomudaspinhao15. pdf. $>$. Acesso em: 26 jul. 2009.

BARROSO, D. Q. Qualidade de mudas de Eucalyptus camaldulensis e E. urophylla produzidas em tubetes e em blocos prensados com diferentes substratos. 1999. Tese (Doutorado em Produção Vegetal) - Universidade Estadual do Norte Fluminense, Goytacazes.

CAMPINHOS JUNIOR, E.; IKEMORI, Y. K. Nova técnica para produção de essências florestais. IPEF, Piracicaba, v. 23, n. 1, p. 47-52, 1983.

CARVALHO FILHO, J. L.; ARRIGONI-BLANK, M. F.; BLANK, F. A. Produção de mudas de angelim (Andira fraxinifolia Benth.) em diferentes ambientes, recipientes e substratos. Ciência Agronômica, Fortaleza, v. 35, n. 1, p. 61-67, 2004.

EMPRESA BRASILEIRA DE PESQUISA AGROPECUÁRIA - EMBRAPA. Centro Nacional de Pesquisa de Solos. Sistema brasileiro de classificação de solos. 2. ed. Rio de Janeiro: Embrapa. 2006. 306 p.

FERRARI, M. P. Cultivo do eucalipto - produção de mudas. Embrapa Florestas, 2003. Disponível em: $<\mathrm{http} / /$ sistemasdeproducao.cnptia.embrapa.br/FontesHTML/ Eucalipto/CultivodoEucalipto/03_03_recipientes.htm.>. Acesso em: 22 jun. 2009.

FERREIRA, M. G. R.; ROCHA, R. B.; SOUZA, V. F. Avaliação do crescimento de mudas de pinhão manso (Jatropha curcas L.) em recipientes de diferentes volumes e acompanhamento do desenvolvimento em campo. 2009. Disponível em: <http://www.cpafro.embrapa.br/media/ arquivos/publicacoes/107_pinhaomanso_revisada.pdf.>. Acesso em: 09 jun. 2012.

GINWAL, H. S.; PHARTYAL, S. S.; RAWAT, P. S.; SRIVASTAVA, R. L. Seed source variation in morphology, germination and seedling growth of Jatropha curcas L. in central India. Silvae Genetica, Krankfurt, v. 54, n. 2, p. 76-80, 2005.

GOMES, J. M.; COUTO, L.; BORGES, R. C. G. Influência do tamanho da embalagem plástica na produção de mudas de Ipê, Copaiba e Angico Vermelho. Revista Árvore, Viçosa, v. 14, n. 1, p. 26-34, 1990.
HÖRING, C. F.; MALAVASI, M. M.; MALAVASI, U. C. Armazenamento não Controlado na qualidade de sementes de Jatropha curcas L. Revista Semina: Ciências Agrárias, Londrina, v. 32, n. 2, p. 521-526, 2011.

JOSÉ, A. C.; DAVIDE, A. C.; OLIVEIRA, S. L. Produção de mudas de aroeira (Schinus terebinthifolius Raddi) para recuperação de áreas degradadas pela mineração de bauxita. Cerne, Lavras, v. 11, n. 2, p. 187-196, 2005.

LANG, A. Efeitos da aplicação de fertilizantes de liberação lenta no estabelecimento de mudas de ipê roxo e angico branco em área de domínio ciliar. 2008. Dissertação (Mestrado em Produção Vegetal) Universidade Estadual do Oeste do Paraná, Marechal Cândido Rondon.

LELES, P. S. S.; CARNEIRO, J. G. A.; BARROSO, D. G.; MORGADO, I. F. Qualidade de mudas de Eucalyptus spp produzidas em blocos prensados e em tubetes. Revista Árvore, Viçosa, v. 24, n. 1, p. 13-20, 2000.

LIMA, R. L. S.; SEVERINO, L. S.; SILVA, M. I. L.; VALE, L. S.; BELTRÃO, N. E. M. Volume de recipiente e composição de substrato para produção de mudas de mamoneira. Ciência Agrotécnica, Lavras, v. 30, n. 3, p. 480-486, 2006.

MALAVASI, U. C.; MALAVASI, M. M. Efeito do volume do tubete no crescimento inicial de plântulas de Cordia trichotoma (Vell.) Arrab. ex Steud e Jacaranda micranta Cham. Ciência Florestal, Santa Maria, v. 16, n. 1, p. 11-16, 2006.

NICOLOSO, F. T.; FORTUNATO, R. P.; ZANCHETTI, F.; CASSOL, L. F.; EISINGER, S. M. Recipientes e substratos na produção de mudas de Maytenus ilicifolia E. Apuleia leiocarpa. Ciência Rural, Santa Maria, v. 30, n. 6, p. 987-992, 2000.

OLIVEIRA, F. Q.; ANDRADRE, L. A.; OLIVEIRA, L. S. B.; MALAQUIAS, J. B.; BEZERRA, F. T. C.; ALMEIDA, D. M. Relações entre ambientes e volumes de recipientes na produção de matéria seca de mudas de Jatropha curcas L. In: CONGRESSO BRASILEIRO DE MAMONA, 4.; SIMPÓSIO INTERNACIONAL DE OLEAGINOSAS ENERGÉTICAS, 1., 2010, João Pessoa. Anais... Campina Grande: Embrapa Algodão, 2010. p. 1468-1472.

PAULINO, J.; FOLEGATTI, M. V.; FLUMIGNAN, D. L.; ZOLIN, C. A.; BARBOZA JUNIOR, C. R. A.; PIEDADE, S. M. S. Crescimento e qualidade de mudas de pinhão manso produzidas em ambiente protegido. Revista Brasileira de Engenharia Agrícola e Ambiental, Campina Grande, v. 15, n. 1, p. 37-46, 2011. 
PEZZUTTI, R. V.; SCNUMACHER, M. V.; HOPPE, J. M. Crescimento de mudas de Eucalyptus globulus em resposta a fertilização. Ciência Florestal, Santa Maria, v. 9, n. 2, p. 117-125, 1999.

SATURNINO, H. M.; PACHECO, D. D.; KAKIDA, J.; TOMINAGA, N.; GONÇALVES, N. P. Cultura do pinhão -manso ( Jatrofa curcas L. ). Informe Agropecuário, Belo Horizonte, v. 26 , n. 229 , p. 44-78, 2005.

SCHIAVO, J. A.; MARTINS, M. A. Produção de mudas de acácia colonizadas com micorrizas e rizóbio em diferentes recipientes. Pesquisa Agropecuária Brasileira, Brasília, v. 38, n. 2, p. 173-178, 2003.

SEVERINO, L. S.; LIMA, R. L. S.; BELTRÃO, N. E. M. Avaliação de mudas de pinhão manso em recipientes de diferentes volumes. Campina Grande: Embrapa Algodão, 2007. 14 p. (Boletim de Pesquisa e Desenvolvimento, 81).

SILVA, P. H. M.; STEIN, L. M. Produção de mudas e recomendações de adubação no viveiro. Piracicaba: IPEF, 2008. Disponível em: <http://www.ipef.br /silvicultura / producaomudas.asp.>. Acesso em: 22 jun. 2009.
SOUZA, P. V. D. Optimización de le produccion de plantones de cítricos en vivero: inoculación com mícorrizas vesiculares arbusculares. 1995. Tesis (Doctoral) - Universidad Politécnica de Valencia, Valencia.

SOUZA, S. L.; FREIRE, M. A. O.; SAMPAIO, L. R. Análise de crescimento inicial do Jatropha curcas em condições de sequeiro. Revista Brasileira de Oleaginosas e Fibrosas, Campina Grande, v. 13, n. 3, p. 99-106, 2009.

TAPANES, N. O.; ARANDA, D. A. G.; CARNEIRO, J. W. de M. Transesterificação dos glicerídeos do óleo de Jatropha curcas L. estudo teórico. Rio de Janeiro: UFF, 2006. Disponível em: <www.biodiesel.gov.br/docs/ congressso2006/producao/Glice27.pdf.> . Acesso em: 22 jul. 2009.

VALE, F. X. R. do; FERNADES FILHO, E. I.; LIBERATO, J. R. Softawe QUANT version 1.0.1. Viçosa, MG: Universidade Federal de Viçosa, abr. 2003.

VIANA, J. S.; GONÇALVES, E. P.; ANDRADE, L. A.; OLIVEIRA, L. S. B.; SILVA, E. O. Crescimento de mudas de Bauhinia forficata Link em diferentes tamanhos de recipientes. Floresta, Curitiba, v. 38, n. 4, p. 663-671, 2008. 